\title{
Where does the drop size distribution come from?
}

\author{
Romain Canu*1, Christophe Dumouchel ${ }^{1}$, Benjamin Duret ${ }^{1}$, Mohamed Essadki², Marc \\ Massot $^{2}$, Thibaut Ménard ${ }^{1}$, Stefano Puggelli ${ }^{3}$, Julien Réveillon ${ }^{1}$, François-Xavier Demoulin ${ }^{1}$ \\ 1UMR 6614-CORIA, Technopôle du Madrillet, B.P. 12, Avenue de I'Université, 76801 Saint \\ Etienne du Rouvray Cedex, France \\ ²Laboratoire EM2C - UPR CNRS 288, Ecole Centrale Paris, Grande Voie des Vignes, 92295 \\ Chatenay-Malabry Cedex, France \\ ${ }^{3}$ Dipartimento di Ingegneria Industriale, Università degli Studi di Firenze, Via S. Marta 3, \\ 50139 Firenze, Italia \\ *Corresponding author: romain.canu@coria.fr
}

\begin{abstract}
This study employs DNS of two-phase flows to enhance primary atomization understanding and modelling to be used in numerical simulation in RANS or LES framework. In particular, the work has been aimed at improving the information on the liquid-gas interface evolution available inside the Eulerian-Lagrangian Spray Atomization (ELSA) framework. Even though this approach has been successful to describe the complete liquid atomization process from the primary region to the dilute spray, major improvements are expected on the establishment of the drop size distribution (DSD). Indeed, the DSD is easily defined once the spray is formed, but its appearance and even the mathematical framework to describe its creation during the initial breakup of the continuous liquid phase in a set of individual liquid parcels is missing. This is the main aim of the present work to review proposals to achieve a continuous description of the DSD formation during the atomization process.

The attention is here focused on the extraction from DNS data of the behaviour of geometrical variable of the liquidgas interface, such as the mean and Gauss surface curvatures. A DNS database on curvature evolution has been generated. A Rayleigh-Plateau instability along a column of liquid is considered to analyse and to verify the capabilities of the code in correctly predicting the curvature distribution. A statistical analysis on the curvatures data, in terms of probability density function, was performed in order to determine the physical parameters that control the curvatures on this test case. Two different methods are presented to compute the curvature distribution and in addition, the probability to be at a given distance of the interface is studied. This approach finally links the new tools proposed to follow the formation of the spray with the pioneering work done on scale distribution analysis.
\end{abstract}

\section{Birth of drop size distribution}

A standard output expected from any atomization model or theory is the drop size distribution (DSD). There are several definitions of this function. Most generally, it is $F_{N}(D)$ in such way that $F_{N}\left(D^{\prime}\right) d D$ is the number of droplet per unit of volume with a diameter $\in\left[D^{\prime}, D^{\prime}+d D[\right.$. In this case, it is the number diameter distribution (NDD) and it may be also normalized to define a probability density function $P_{N}(D)$.

The function $F_{N}(D)$ requires the possibility to count the number of droplets. Thus, it is necessary to separate the liquid phase in a set of discrete elements. Usual atomization starts with a continuous liquid flow (for instance a liquid jet) and during the atomization process the splitting of the continuous liquid phase occurs. This phase of the atomization process can be associated to the so-called primary breakup. Once it is created, for fixed external conditions and generally considering the whole spray, the NDD may evolve towards an asymptotic state, for which numerous theoretical and experimental works are reported in the literature [1]. To address more complex situations or to determine its function in space and time, a transport equation for the DSD is required and in the case of the $F_{N}(D)$ this is the Williams-Boltzmann equation (WBE) [2].

Although the flow inside the injector as well as surface instabilities and the way to solve the WBE have been the subject of important researches, few works deal with the droplet generation step to estimate the initial DSD. One reason is the lack of data to justify any proposal and the other reason is the non-applicability of the NDD definition during the primary breakup. Indeed, considering a liquid jet before the detachment of a liquid parcel, the notion of a diameter cannot be reasonably introduced. However, considering an initial instability over a liquid surface, such unsteadiness will evolve in time and create wrinkles at a certain length scale that should be in some way related on a theoretical point of view to the diameter of the droplet that will be then generated. It is clear that a link between these two subsequent steps is still missing. Regarding the data in this transition zone during primary breakup, there have been great progresses both in experimental techniques and in numerical simulations. 
The main goal of the present study is therefore the proposition of an innovative extended definition of the drop size distribution to be used all along the atomization process, leading to the so called Surface Curvature Distribution (SDF). The definition of the SDF will be first introduced and related to some characteristic geometrical properties of the liquid/gas interface, such as the mean and Gauss curvatures. Then, numerical simulations will be then used to extract its evolution on a fundamental breakup test case. The well-known Archer code [3], [4], where a combined VOF-Level set approach is used to capture the interface and a ghost-fluid method is applied to represent accurately the jump of variable across the liquid-gas interface, has been used to this end.

The paper is structured as follows: the next section is devoted to the description of the SDF and to the introduction of a relation between the SDF and the NDF for a spherical droplet based on surface curvatures. In the second part of the paper, a Rayleigh-Plateau test case has been considered to determine the evolution of the two curvatures to show how the breakup process from a liquid jet to the final droplet can be directly described employing such surface geometrical properties.

\section{An extended definition of the drop size distribution}

The purpose of this section is to propose an extended definition of the drop size distribution that may applicable all along the atomization process including the primary breakup zone. Before looking at the DSD definition, it should be pointed out that the same problem appears to define a mean diameter: it is a moment of the NDD and therefore it can be defined only once the continuous liquid jet has been broken in a set of liquid droplets. From the pioneering work of Vallet and Borghi [5], [6] it is known that the definition of the mean diameter can be replace beneficially by the more general mean surface density $\Sigma$, that is the surface area per unit of volume. This quantity can be defined everywhere whatever is the liquid phase topology and, combined with the liquid volume fraction $\alpha$, it gives the mean Sauter diameter once the spray is formed: $D_{32}=\frac{6 \alpha}{\Sigma}$. Recently, Essadki et al [7] used high order fractional moments of the DSD for disperse phase, where the size is given by the surface area of droplet, to recover some interface geometrical quantities already used in describing the gas-liquid interface in [9]. These quantities are the volume fraction, the mean surface density and the two averaged Gauss $G=\kappa_{1} * \kappa_{2}$ and mean $H=\frac{\kappa_{1}+\kappa_{2}}{2}$ curvatures, where $\kappa_{1}$ and $\kappa_{2}$ are the two principal curvatures of the surface.

To overcome the limits of the DSD in modelling a gas-liquid interface, some surface characteristics can be used since they should carry the information of the DSD which is hidden during the wrinkling process.

The proposal here is to look at the curvature $(\kappa)$ distribution along the liquid-gas surface. In this case the distribution of curvature is $F_{S}(\kappa)$ and the $F_{S}\left(\kappa^{\prime}\right) d \kappa$ is the area of surface with a curvature $\kappa \in\left[\kappa^{\prime}, \kappa^{\prime}+d \kappa[\right.$. This leads to the definition of a surface curvature distribution (SCD). However, an accurate description of the interface cannot be restricted to one geometrical variable as it is often used in the DSD for disperse phase. Therefore, we use the Gauss and mean curvatures as phase-space variable, leading to a bi-variate $\operatorname{SCD}: F_{S}(H, G)$. Once the spray is formed, the DSD is a particular case of $F_{S}(H, G)$ since for spherical droplets $\kappa_{1}=\kappa_{2}=2 / D$.

Indeed, for a disperse phase of spherical droplets, the Gauss curvature can be simply expressed as $G=4 \pi / S$, where $S=\pi D^{2}$ is the droplet surface area. Therefore, the two functions $4 \pi / G F_{S}(H=\sqrt{G}, G)$ and $F_{D}(D)$ are equivalents, since they count the probable number of droplets per volume and per radius or Gauss curvature. The link between the two distributions has been already generalized in [8] for non-spherical droplets by using some interesting topological properties of Gauss curvature.

Therefore, considering that during primary atomization the liquid-gas interface undergoes different instabilities and deformation, the interface state can be described geometrically by the local curvatures. When the curvatures reach high values, the liquid breakup mechanism is activated and lead to generate droplets of diameter related to the curvatures of the previous interface state. Hence, the first proposal has been to carry the curvature distribution by the surface. However, any spray may be characterised by a volumetric distribution. For a spray, the DSD becomes $F_{V}(D)$ in such way that $F_{V}\left(D^{\prime}\right) d D$ is the liquid volume fraction of droplets with a diameter $\in\left[D^{\prime}, D^{\prime}+d D[\right.$. This volumetric distribution can be also extended to any liquid topology, leading to the volumetric distance function distribution $(\mathrm{V} \varphi \mathrm{D})$, that can be a second proposal. It is defined by $F_{V}(\varphi)$ in such way that $F_{V}\left(\varphi^{\prime}\right) d \varphi$ is the volume fraction of flow that is at a distance $\varphi$ from the interface, with $\varphi \in\left[\varphi^{\prime}, \varphi^{\prime}+d \varphi[, \varphi\right.$ is positive in the liquid phase and negative in the gas phase. With this definition the following relation stands:

The gas volume fraction is $\alpha_{g}=\int_{-\infty}^{0} F_{V}(\varphi) d \varphi$, the liquid volume fraction is $\alpha_{l}=\int_{0}^{+\infty} F_{V}(\varphi) d \varphi$, and the mean surface density $\Sigma=F_{V}(0)$.

The $\mathrm{V} \varphi \mathrm{D}$ gives the distribution of the flow with respect to the distance to the interface. The link with the DSD is not straightforward. However, this distribution can be clearly identified on a given geometric object and in particular for a spherical droplet. Indeed, this idea has been explored by Dumouchel and co-workers [12,13] with the concept of scale distribution, $E_{3}(d)$. The definition of this function for a given object (for instance a droplet) is based on the total object volume $V_{0}$ and the volume $V(d)$ defined by all points at a distance $d$ or greater to the object surface. 
The scale distribution is given by $E_{3}(d)=\frac{\left(V_{0}-V(d)\right)}{V_{0}}$. The link between the scale distribution and the $\mathrm{V} \varphi \mathrm{D}$ can be express readily by $E_{3}(d)=\frac{F_{V}(\varphi=d)}{\alpha_{l}}$. Dumouchel et al. have worked on experimental data in 2D [12] and more recently they have used 3D simulation (ICM + DNS) to extract 3D results [13]. A link must exist between the scale distribution or the volume fraction distribution with the curvature or at least an average curvature of the surface. This point is still under investigation inside our research group.

\section{First application of the definitions}

As a first step a very simple configuration corresponding to a Rayleigh-Plateau instability, where a set of droplet is created from an initial column of liquid, is here used to show how the surface curvatures and $\mathrm{V} \varphi \mathrm{D}$ can be used to describe a breakup event. This is a standard test case for the code Archer that is known to give good results on this configuration. Two methods have been used to extract the curvature distribution. The first one is based on the distance function that is part of the numerical procedure in the resolution of the two-phase flow in Archer code. The normal to the surface $\vec{n}=-\frac{\vec{\nabla} \varphi}{|\vec{\nabla} \varphi|}$ is first calculated. Then, the matrix $P=I-\vec{n} \vec{n}^{T}$ with $I$ the identity matrix, and the Hessian matrix $H m=\frac{\partial^{2} \varphi}{\partial x_{i} \partial x_{j}}$ are calculated in order to have the matrix $G m=-\frac{P H m P}{|\vec{\nabla} \varphi|}$. Finally, the two principal curvatures $\kappa_{1}$ and $\kappa_{2}$ are obtained by :

$\kappa_{1}=\frac{T+\sqrt{2 F^{2}-T^{2}}}{2}$ and $\kappa_{2}=\frac{T-\sqrt{2 F^{2}-T^{2}}}{2}$

with $T$, the trace of the matrix $G m$ and $F$, the Frobenius norm of the matrix $G m$. For a more detailed explanation of the method, see [11]. In Archer, these curvatures are calculated on the center of the mesh cells, so in order to have the right curvatures on the interface, the curvature radius is corrected by the distance function which gives the distance from the center of the cell to the interface.

The second one has been developed in [8], where the gas-liquid interface is discretized with a 2D triangulated mesh using the Marching Cube algorithm. The curvature is computed at each vertex of the created mesh, as spatial averages around this vertex, based on some results of discrete differential geometry [10]. Then, in order to eliminate the noise of the estimated curvatures, the authors of [8] used an adequate spatial-averaging process, which preserves some topological feature of the gas-liquid interface such as computing the droplet number from the bivariate SCD: $F_{S}(H, G)$, while filtering the noise.

Finally, the first result on the $\mathrm{V} \varphi \mathrm{D}$ have been obtained through the distance function. All these concepts have been explored in a simple test case described in the next section before addressing more complex test cases up to the final atomization.

\section{Numerical Results}

The validation case used here is the Rayleigh-Plateau instability, which consists on the formation of droplets from an initial liquid jet due to surface tension with the surrounding gas. We computed a quarter of a cylinder and used symmetric boundary conditions in a computational box of $1,5.10^{-4} \times 1,0.10^{-4} \times 1,0.10^{-4} \mathrm{~m}$ with a $96 \times 64 \times 64$ mesh. The liquid and gas properties are the following ones:

$\rho_{l}=1000 \mathrm{~kg} \cdot \mathrm{m}^{-3} \quad \rho_{g}=1 \mathrm{~kg} \cdot \mathrm{m}^{-3}$

$\mu_{l}=1,0 \cdot 10^{-3} \mathrm{~kg} \cdot \mathrm{m}^{-1} \cdot \mathrm{s}^{-1} \quad \mu_{g}=1,879 \cdot 10^{-5} \mathrm{~kg} \cdot \mathrm{m}^{-1} \cdot \mathrm{s}^{-1}$

$\sigma=0,072 \mathrm{~kg} \cdot \mathrm{s}^{-2}$

The cylinder has a radius $R=3,34 \cdot 10^{-5} \mathrm{~m}$ and the initial perturbation, essential to observe the instability, has an amplitude of $10 \%$ of the radius of the cylinder and a wavelength of $3.10^{-4} \mathrm{~m}$ (twice the length of the box). The initial conditions have been chosen in order to have a wave number satisfying $k R=0,7$ which correspond to the fastest growth rate. The two previous methods to extract the curvature distribution were tested (Figure 1) but no significant differences were observed, which shows that this case is well resolved with a high mesh resolution.

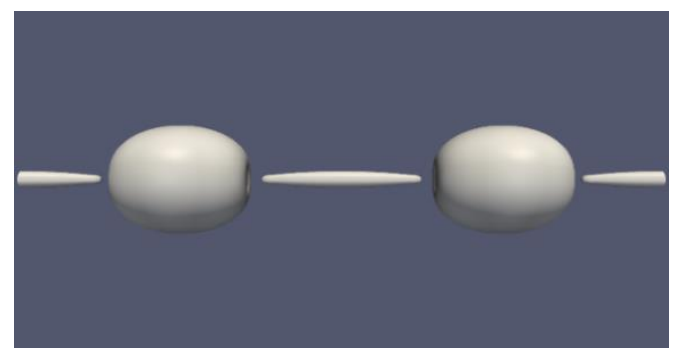



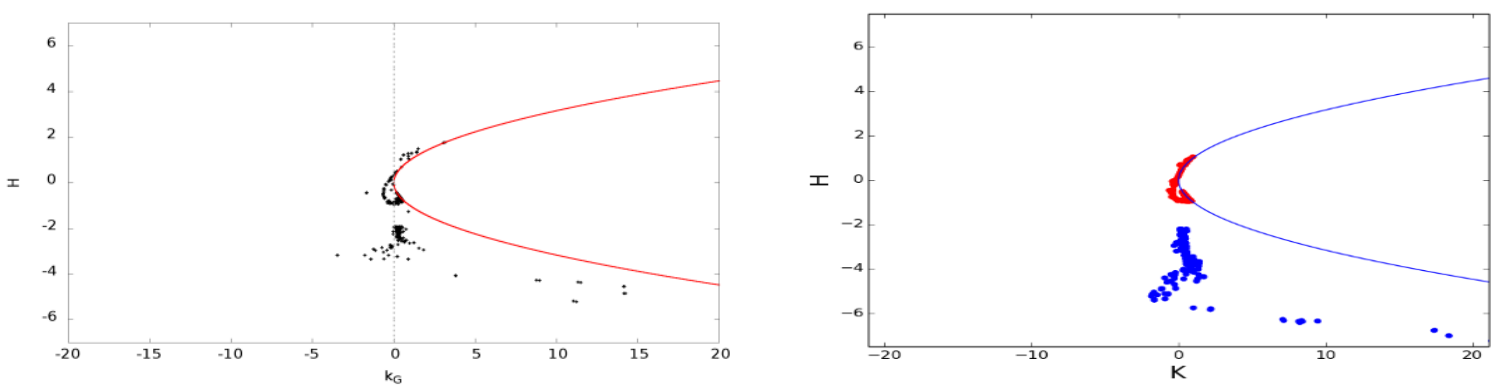

Figure 1. Top left : Rayleigh Plateau instability at $t=1,88.10^{-4} \mathrm{~s}$; Bottom left : Mean curvature in function of Gauss curvature with the first method (red curve represents $\kappa_{1}=\kappa_{2}$ and the dotted line represents $G=0$ ) ; Bottom right : Mean curvature in function of Gauss curvature with the second method (blue curve represents $\kappa_{1}=\kappa_{2}$, red points represents the right part of the simulation and blue points, the left part)

In the following figures, the mean and total curvatures are provided as dimensionless using the radius of the initial cylinder as reference length, whereas for the Gauss curvature the radius squared has been used to this end. The mean curvature of a sphere with a radius of $d x$ is 21,4 and its Gauss curvature is 457,5 .

On Figures 6 and 7, the distance function $\varphi$ is reported as dimensionless using $d x$ as reference and the curves are shown between $-l_{x}$ and $l_{x}$. The same validation case is used in these figures but with a $36 \times 24 \times 24$ mesh sizing. The surface evolution of the Rayleigh-Plateau instability in different four time steps is shown in Figure 2, where it is possible to see the initial configuration ( $t a$ ), the deformation of the cylinder $(t b)$, the moment of the break-up (tc) and the final droplet's formation (td). It is now interesting to analyse the evolution of surface curvatures during this breakup process.

It should be pointed out that when the cylinder is weakly deformed (Figure 6), in terms of PDF profile we are very close to the theoretical profile of a cylinder with a linear curve inside the liquid part. For the total curvature (Figure 5 left) and Gauss curvature (Figure 4 left) cumulative, a step profile at values corresponding to this cylinder is obtained. Furthermore, on the graph showing mean curvature in function of Gauss curvature (Figure 3 top left), all the points are around the vertical line representing the cylinder.

Then, when such cylinder is deformed (tb), we observe negative values for Gauss curvature (Figure 4 right) corresponding to all the saddle points in the middle of the cylinder. This portion of cylinder is visible on the mean and Gauss curvatures graph (Figure 3 top right) and it is the arc formed by the points. Besides, on this graph, the points, which represent the big part of the cylinder, get closer to the red curve corresponding to a sphere.

When the break-up happens ( $t c$ ), the previous arc breaks up and two sets of points appear (Figure 3 bottom left), one of which is located on the red curve. These points correspond to the big droplet. Indeed, the mean curvature is approximately 0.5 and the Gauss curvature about 0.25 which correspond to a radius twice as big as the initial jet radius. The other points, located below the red curve, correspond to the small ellipsoid. Here, the mean curvature is quite high because of the small thickness and the Gauss curvature is weaker because the curvature is close to zero along the length. The big droplet is characterised by the step on the total curvature cumulative (Figure 5 right) and the small ellipsoid by all the high negative values. It should be pointed out that such break-up process is mainly evident on the curvature plot since small variations on the interface geometry can lead strong changes in the curvature values.

Finally, when the two droplets are formed (td), for the mean and Gauss curvatures graph (Figure 3 bottom right) most of the points are on the red curve, which represents the spheres. The points corresponding to the big droplet are located approximately at the same place than the previous time. The points corresponding to the small droplet are those having an higher curvature because of, obviously, its small radius. On Figure 7, PDF profile becomes parabolic in the liquid part, as expected for spheres, with higher values of the distance function because the biggest droplet is bigger than the initial cylinder. 

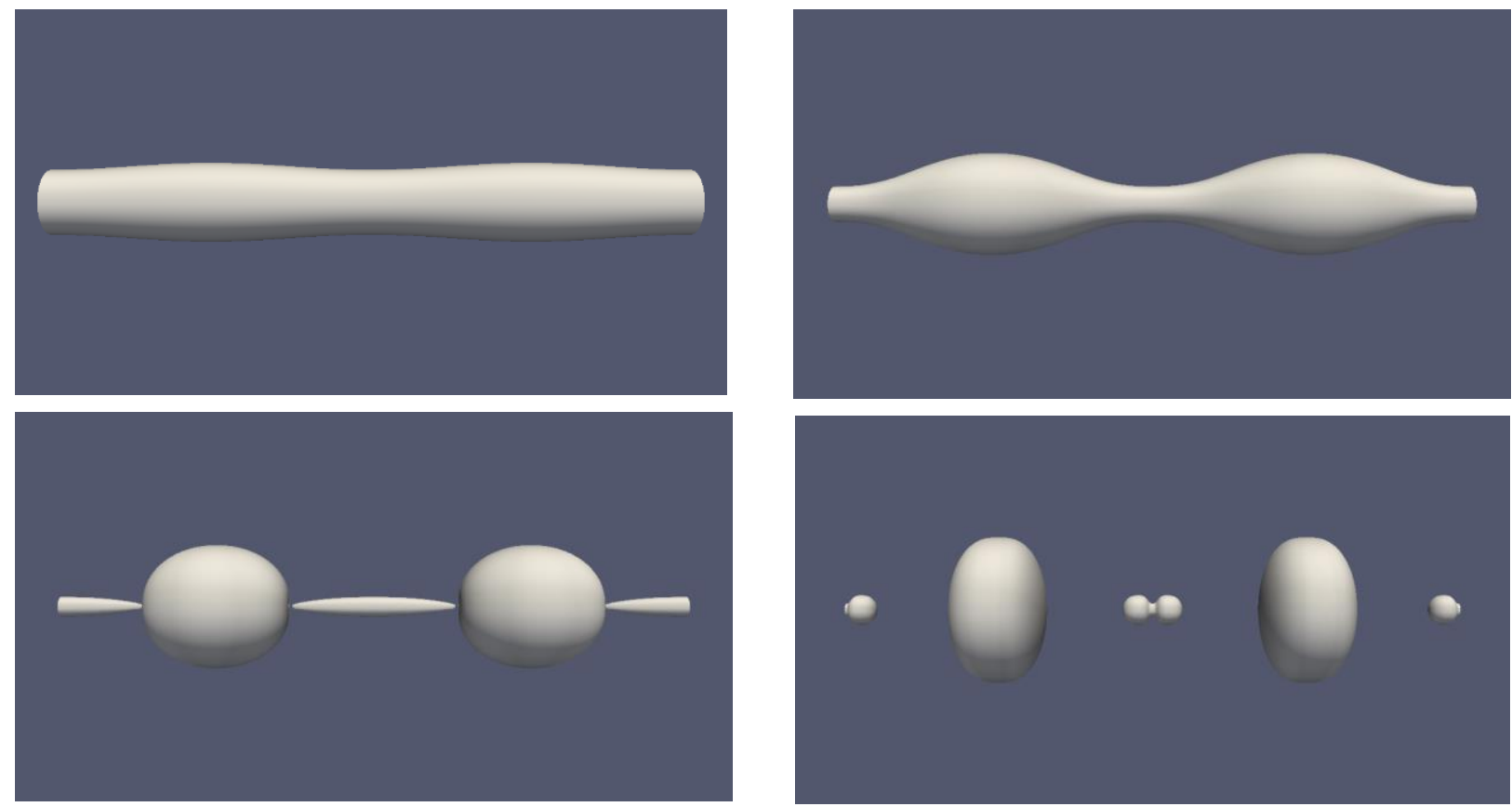

Figure 2. Top left : Rayleigh Plateau instability at $t_{a}=3,92 \cdot 10^{-7} \mathrm{~s}$; Top right : Rayleigh Plateau instability at $t_{b}=1,49.10^{-4} \mathrm{~s}$; Bottom left : Rayleigh Plateau instability at $t_{c}=1,86.10^{-4} \mathrm{~s}$; Bottom right : Rayleigh Plateau instability at $t_{d}=2,03.10^{-4} \mathrm{~s}$
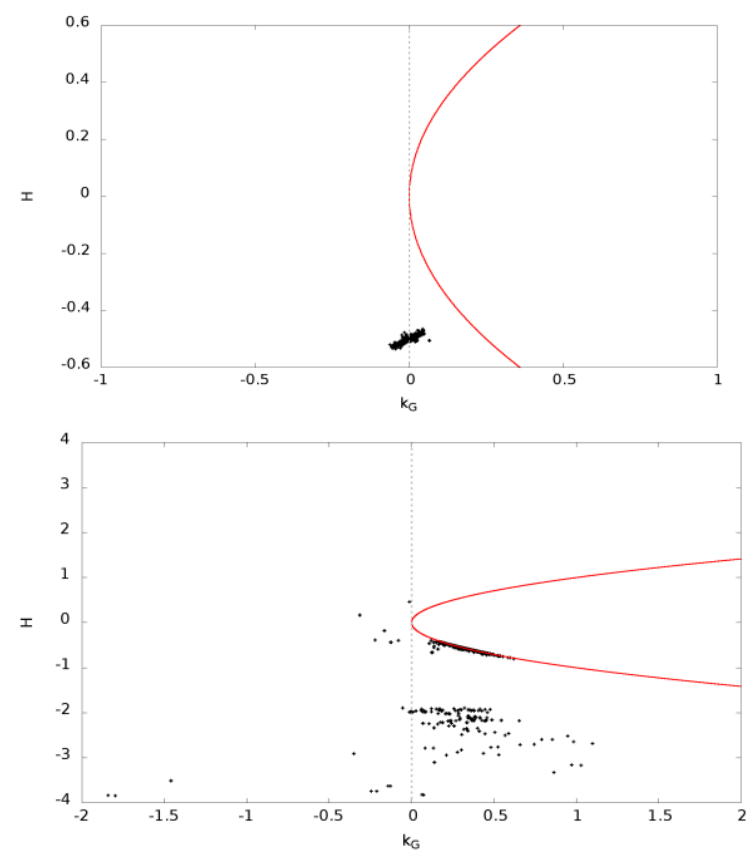
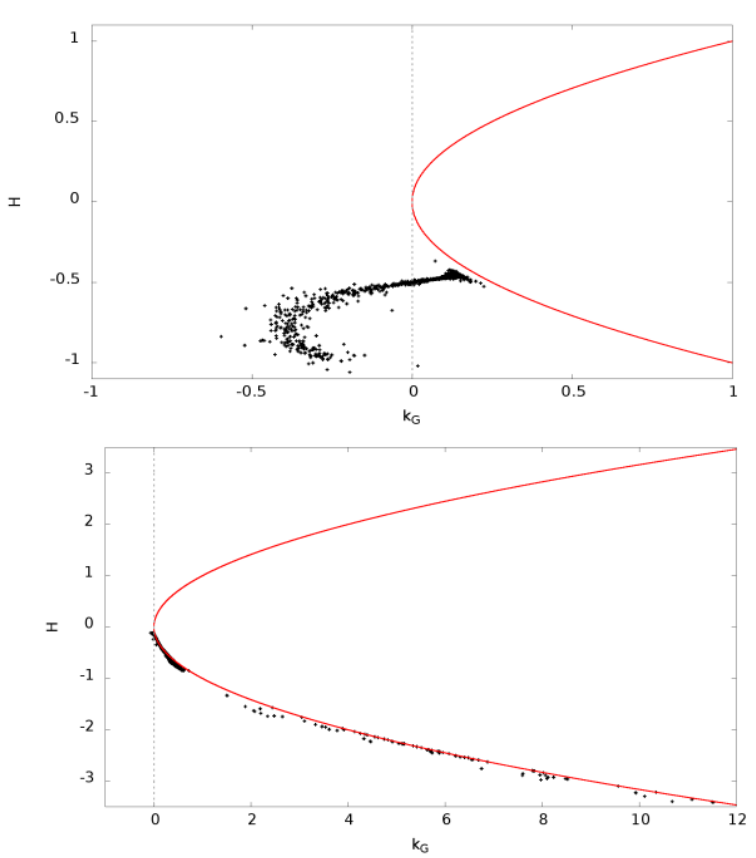

Figure 3. Mean curvature in function of Gauss curvature (Top left : at $t_{a}$; Top right : at $t_{b}$; Bottom left : at $t_{c}$; Bottom right : at $t_{d}$ (red curve represents $\kappa_{1}=\kappa_{2}$ so a sphere and the dotted line represents $G=0$ so a cylinder) 

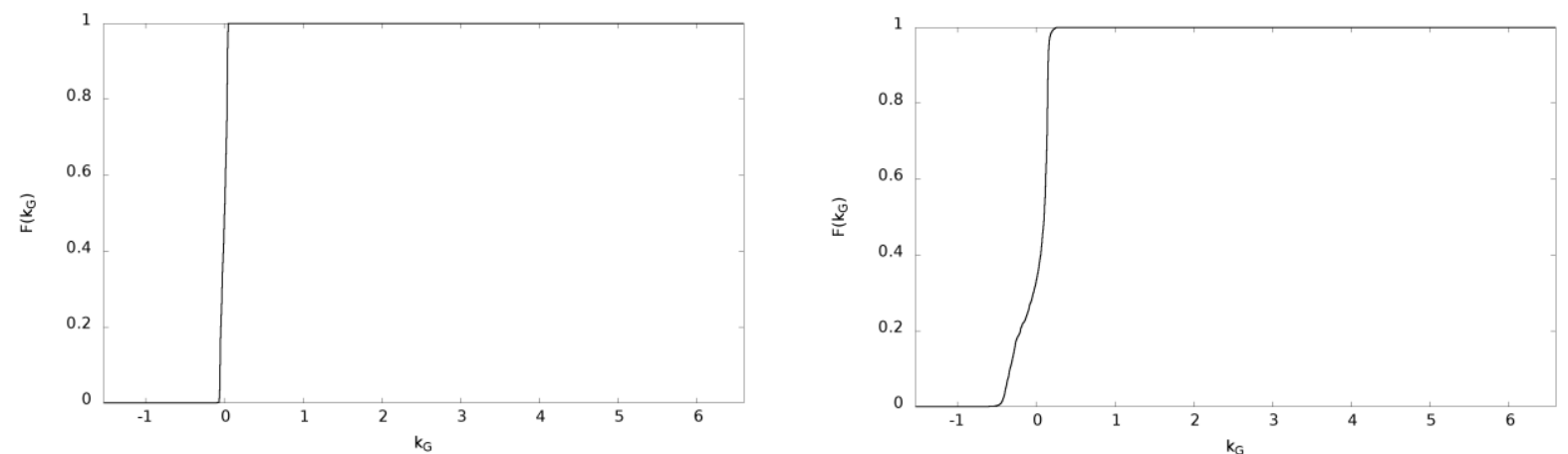

Figure 4. Cumulative of the Gauss curvature (Left : at $t_{a} ;$ Right : at $t_{b}$ )
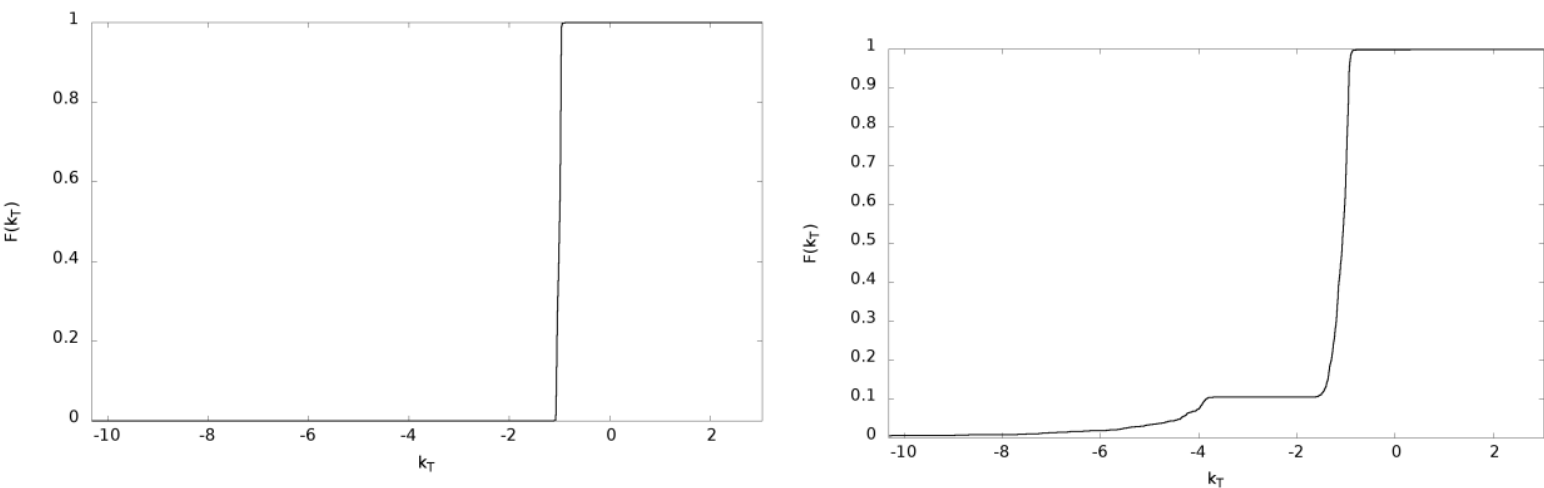

Figure 5. Cumulative of the total curvature (Left : at $t_{a}$; Right: at $t_{c}$ )
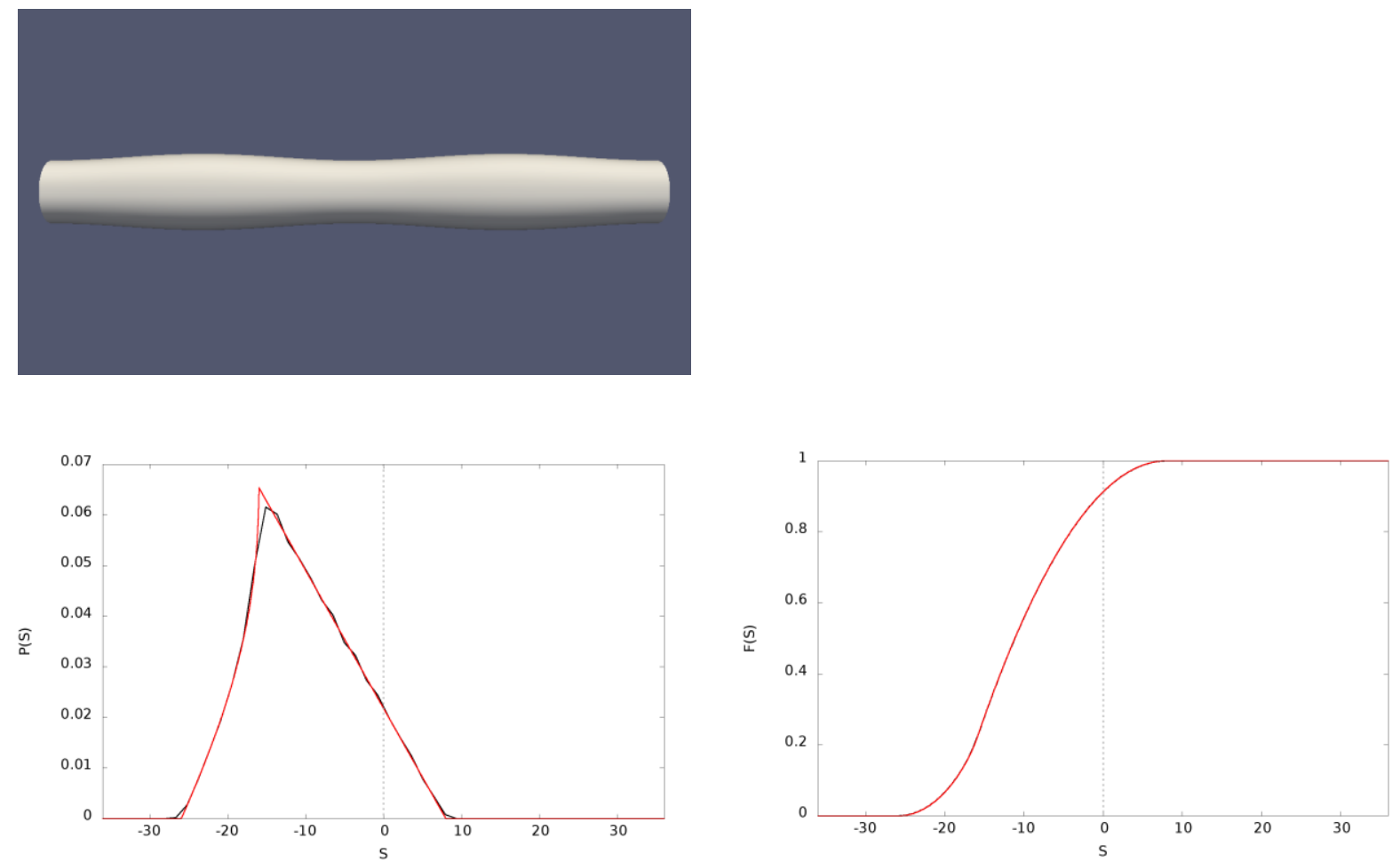

Figure 6. Top left : Rayleigh Plateau instability at $t_{a}$; Bottom left : pdf of the distance function (red curve represents the initial pdf profile for a cylinder) ; Bottom right : cumulative of the distance function (red curve represents the initial cumulative profile for a cylinder) ; the dotted line separates liquid (positive values) and gas (negative values) 

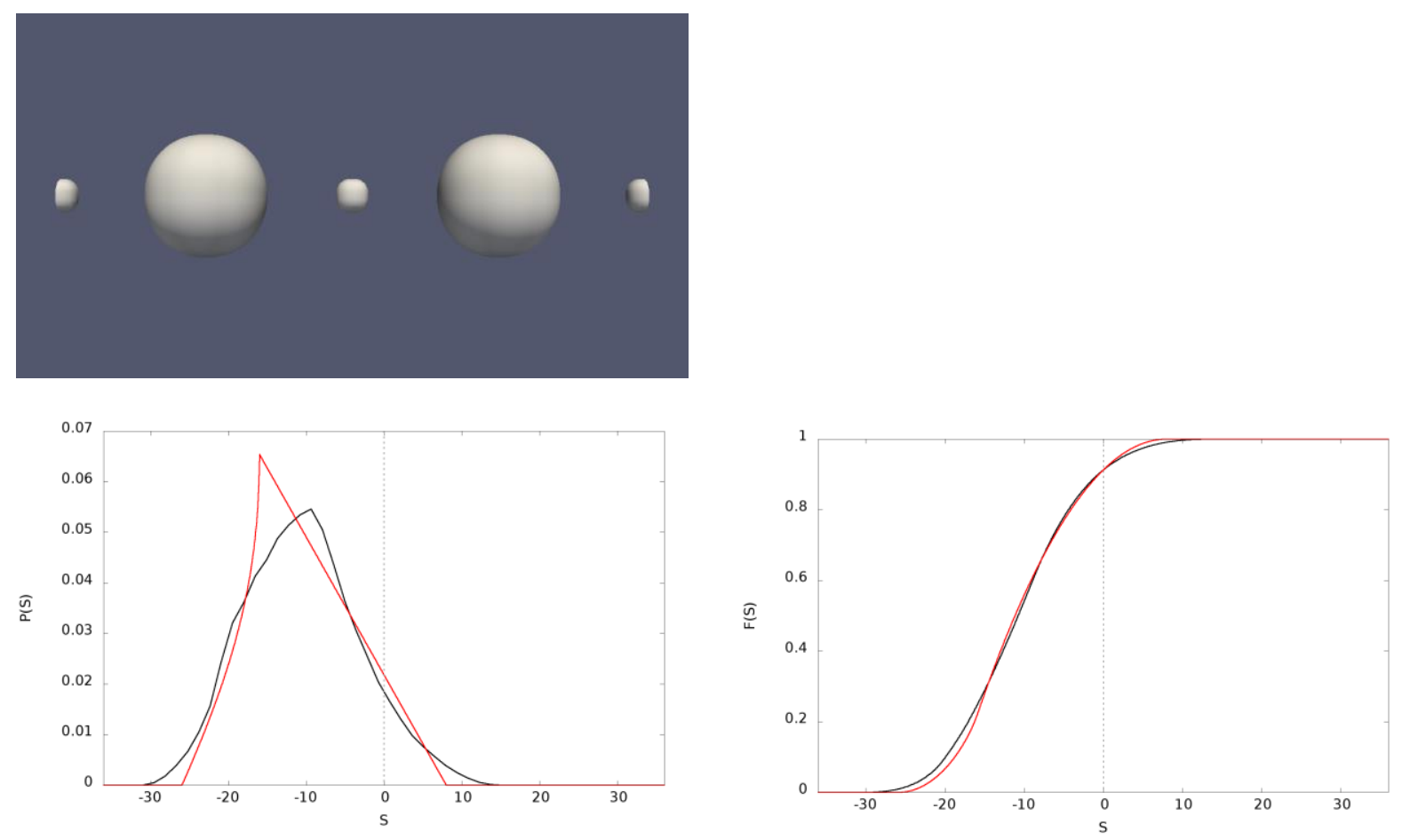

Figure 7. Top left : Rayleigh Plateau instability at $t_{d}$; Bottom left : pdf of the distance function (red curve represents the initial pdf profile for a cylinder) ; Bottom right : cumulative of the distance function (red curve represents the initial cumulative profile for a cylinder) ; the dotted line separates liquid (positive values) and gas (negative values)

\section{Conclusions}

This study shows how DNS of two-phase flows can be employed to improve understanding and modelling of primary atomization to be then used in RANS or LES framework. In particular, the study is aimed at enhancing the information available in ELSA framework through the introduction of liquid/gas interface curvatures. Firstly, on a mathematical point of view, an extended definition of the drop size distribution to be used all along the atomization process is proposed, leading to the so called surface curvature distribution. Then, the extraction of surface curvatures is shown for a simple Rayleigh-Plateau test case before to go on with more complex and computationally costly tests. Two different methods to extract the curvature distributions are tested, even if they do not here determine appreciable differences. The evolution of Gauss and mean curvatures is analysed in detail in order to show how the breakup process from a liquid jet to the final droplet can be easily described in terms of such surface geometrical properties.

\section{Nomenclature}

$\begin{array}{ll}\text { D } & \text { Droplet diameter [m] } \\ \text { G } & \text { Gauss curvature }[\mathrm{m}-2] \\ \text { H } & \text { Mean curvature }[\mathrm{m}-1] \\ \text { R } & \text { Cylinder radius }[\mathrm{m}]\end{array}$

\section{Greek}

$\begin{array}{ll}\alpha & \text { Liquid volume fraction [-] } \\ \Sigma & \text { Mean surface density [m-1] } \\ \kappa & \text { Principal curvature [m-1] } \\ \varphi & \text { Distance function from the interface [m] } \\ \rho & \text { Density [kgm-3] }\end{array}$


$\begin{array}{ll}\mu & \text { Viscosity [kgm-1 s-1] } \\ \sigma & \text { Surface tension [kgs-2] }\end{array}$

\section{Subscripts}

$1 \quad$ Liquid

g Gas

\section{Acronyms}

DNS Direct Numerical Simulation

DSD Drop Size Distribution

ICM Interface Capturing Method

LES Large Eddy Simulation

NDD Number Diameter Distribution

RANS Reynolds Average Navier Stokes

SCD Surface Curvature Distribution

$\mathrm{V} \varphi \mathrm{D} \quad$ Volumetric distance function Distribution

\section{References}

[1] E. Babinsky and P. E. Sojka, "Modeling drop size distributions," Prog. Energy Combust. Sci., vol. 28, no. 4, pp. 303-329, 2002.

[2] F. Williams, "Spray Combustion and Atomization," Phys. Fluids, vol. 1, no. 6, pp. 541-545, 1958.

[3] S. Tanguy and A. Berlemont, "Application of a level set method for simulation of droplet collisions," Int. J. Multiph. Flow, vol. 31, no. 9, pp. 1015-1035, 2005.

[4] T. Menard, S. Tanguy, and A. Berlemont, "Coupling level set/VOF/ghost fluid methods: Validation and application to 3D simulation of the primary break-up of a liquid jet," Int. J. Multiph. Flow, vol. 33, no. 5, pp. 510-524, 2007.

[5] A. Vallet and R. Borghi, "Modélisation Eulerienne de L'atomisation d'un Jet Liquide," C R Acad Sci Paris Sér II $B$, vol. 327, pp. 1015-1020, 1999.

[6] A. Vallet, A. A. Burluka, and R. Borghi, "Development of a Eulerian model for the 'Atomization' of a liquid jet," At. Sprays, vol. 11, no. 6, pp. 619-642, 2001.

[7] M. Essadki, S. de Chaisemartin, F. Laurent, and M. Massot, "High Order Moment Model for Polydisperse Evaporating Sprays Towards Interfacial Geometry," submitted to SIAM J. Appl. Math, (2016). Available on HAL, https://arxiv.org/pdf/1608.07148

[8] M. Essadki, F. Drui, S. de Chaisemartin, A. Larat, T. Ménard and M. Massot, "Statistical modeling of the gasliquid interface: Toward a unified description of the disperse and separated phases flows," submitted to IJMF, (2017) pp1--25. Available on HAL

[9] M. Essadki, S. de Chaisemartin, M. Massot, F. Laurent, A. Larat, S. Jay, "Adaptive mesh refinement and high order geometrical moment method for the simulation of polydisperse evaporating sprays," Oil \& Gas Science and Technology, vol. 71, no. 5, (2016), 1-25

[10] M. Meyer, M. Desbrun, P. Schröder, A. H. Barr, "Discrete differential-geometry operators for triangulated 2manifolds." Visualization and Mathematics III pp 35-57 (2001) 1-26.

[11] G. Kindlmann, R. Whitaker, T. Tasdizen, T. Möller, "Curvature-based transfer functions for direct volume rendering : Methods and applications" In Proc. of IEEE Visualization 2003 (2003), pp. 513-520.

[12] C. Dumouchel, T. Ménard, W. Aniszewski, "Towards an Interpretation of the scale Diffusivity in Liquid Atomization Process : An Experimental Approach," Physica A, vol. 438, 612-624, 2015

[13] C. Dumouchel, W. Aniszewski, T. T. Vu, T. Ménard, (2017) International Journal of Multiphase Flow, DOI : 10.1016/j.jimultiphaseflow.2017.03.012 